\title{
The Partial Averaging of Fuzzy Differential Inclusions on Finite Interval
}

\author{
Andrej V. Plotnikov ${ }^{1}$ and Tatyana A. Komleva ${ }^{2}$ \\ ${ }^{1}$ Department of Applied Mathematics, Odessa State Academy of Civil Engineering and Architecture, Didrihsona Street 4, \\ Odessa 65029, Ukraine \\ ${ }^{2}$ Department of Mathematics, Odessa State Academy of Civil Engineering and Architecture, Didrihsona Street 4, \\ Odessa 65029, Ukraine
}

Correspondence should be addressed to Andrej V. Plotnikov; a-plotnikov@ukr.net

Received 12 February 2014; Accepted 23 April 2014; Published 4 May 2014

Academic Editor: Nikolai N. Leonenko

Copyright (c) 2014 A. V. Plotnikov and T. A. Komleva. This is an open access article distributed under the Creative Commons Attribution License, which permits unrestricted use, distribution, and reproduction in any medium, provided the original work is properly cited.

The substantiation of a possibility of application of partial averaging method on finite interval for differential inclusions with the fuzzy right-hand side with a small parameter is considered.

\section{Introduction}

In 1990, Aubin [1] and Baidosov [2, 3] introduced differential inclusions with the fuzzy right-hand side. Their approach is based on usual differential inclusions. In 1995, Hüllermeier [4-6] introduced the concept of $R$-solution similar to how it has been done in [7]. Further in [8-20], various properties of solutions of fuzzy differential inclusions and their applications at modeling of various natural-science processes were considered.

The averaging methods combined with the asymptotic representations (in Poincare sense) began to be applied as the basic constructive tool for solving the complicated problems of analytical dynamics described by the differential equations. After the systematic researches done by N. M. Krylov, N. N. Bogoliubov, Yu. A. Mitropolsky, and so forth, in 1930s, the averaging method gradually became one of the classical methods in analyzing nonlinear oscillations.

In works [21,22], the possibility of application of schemes of full and partial averaging for differential inclusions with the fuzzy right-hand side, containing a small parameter, was proved. By proving these theorems, the scheme offered by Plotnikov et al. for a substantiation of schemes of an average of usual differential inclusions [23-28] was used. In this work, the possibility of application of partial averaging method for fuzzy differential inclusions without passage to reviewing of separate solutions is proved; that is, all estimations are spent for $R$-solution corresponding fuzzy systems.

\section{Preliminaries}

Let $\operatorname{comp}\left(R^{n}\right)\left(\operatorname{conv}\left(R^{n}\right)\right)$ be a family of all nonempty (convex) compact subsets from the space $R^{n}$ with the Hausdorff metric:

$$
h(A, B)=\min _{r \geq 0}\left\{S_{r}(A) \supset B, S_{r}(B) \supset A\right\},
$$

where $A, B \in \operatorname{comp}\left(R^{n}\right)$ and $S_{r}(A)$ is $r$-neighborhood of set A.

Let $E^{n}$ be a family of all $u: R^{n} \rightarrow[0,1]$ such that $u$ satisfies the following conditions:

(1) $u$ is normal; that is, there exists an $x_{0} \in R^{n}$ such that $u\left(x_{0}\right)=1$;

(2) $u$ is fuzzy convex; that is,

$$
u(\lambda x+(1-\lambda) y) \geq \min \{u(x), u(y)\},
$$

for any $x, y \in R^{n}$ and $0 \leq \lambda \leq 1$; 
(3) $u$ is upper semicontinuous; that is, for any $x_{0} \in R^{n}$ and $\varepsilon>0$ exists $\delta\left(x_{0}, \varepsilon\right)>0$ such that $u(x)<u\left(x_{0}\right)+\varepsilon$ whenever $\left\|x-x_{0}\right\|<\delta\left(x_{0}, \varepsilon\right), x \in R^{n}$;

(4) the closure of the set $\left\{x \in R^{n}: u(x)>0\right\}$ is compact.

If $u \in E^{n}$, then $u$ is called a fuzzy number and $E^{n}$ is said to be a fuzzy number space.

Definition 1. The set $\left\{x \in R^{n}: u(x) \geq \alpha\right\}$ is called the $\alpha$-level $[u]^{\alpha}$ of a fuzzy number $u \in E^{n}$, for $0<\alpha \leq 1$. The closure of the set $\left\{x \in R^{n}: u(x)>0\right\}$ is called the 0 -level $[u]^{0}$ of a fuzzy number $u \in E^{n}$.

It is clear that the set $[u]^{\alpha} \in \operatorname{conv}\left(R^{n}\right)$, for all $0 \leq \alpha \leq 1$.

Theorem 2 (see [29] (stacking theorem)). If $u \in E^{n}$, then

(1) $[u]^{\alpha} \in \operatorname{conv}\left(R^{n}\right)$, for all $\alpha \in[0,1]$;

(2) $[u]^{\alpha_{2}} \subset[u]^{\alpha_{1}}$, for all $0 \leq \alpha_{1} \leq \alpha_{2} \leq 1$;

(3) if $\left\{\alpha_{k}\right\}$ is a nondecreasing sequence converging to $\alpha>0$, then $[u]^{\alpha}=\bigcap_{k \geq 1}[u]^{\alpha}$.

Conversely, if $\left\{A_{\alpha}: \alpha \in[0,1]\right\}$ is the family of subsets of $R^{n}$ satisfying conditions (1)-(3), then there exists $u \in E^{n}$ such that $[u]^{\alpha}=A_{\alpha}$ for $0<\alpha \leq 1$ and $[u]^{0}=\overline{\bigcup_{0<\alpha \leq 1} A_{\alpha}} \subset A_{0}$.

Let $\theta$ be the fuzzy number defined by $\theta(x)=0$, if $x \neq 0$ and $\theta(0)=1$.

Define $D: E^{n} \times E^{n} \rightarrow[0, \infty)$ by the relation

$$
D(u, v)=\sup _{0 \leq \alpha \leq 1} h\left([u]^{\alpha},[v]^{\alpha}\right) \text {. }
$$

Then, $D$ is a metric in $E^{n}$. Further, we know that [30]

(i) $\left(E^{n}, D\right)$ is a complete metric space;

(ii) $D(u+w, v+w)=D(u, v)$, for all $u, v, w \in E^{n}$;

(iii) $D(\lambda u, \lambda v)=|\lambda| D(u, v)$, for all $u, v \in E^{n}$ and $\lambda \in R$.

\section{Fuzzy Differential Inclusion: $R$-Solution}

Consider the fuzzy differential inclusion

$$
\dot{x} \in F(t, x), \quad x(0) \in X_{0},
$$

where $x \in R^{n}, t \in[0, T] \subset R_{+}, F:[0, T] \times R^{n} \rightarrow E^{n}, X_{0} \in E^{n}$.

We interpret (4) as a family of differential inclusions (see $[7,9,10])$ :

$$
\dot{x}_{\alpha} \in\left[F\left(t, x_{\alpha}\right)\right]^{\alpha}, \quad x_{\alpha}(0) \in\left[X_{0}\right]^{\alpha}, \quad \alpha \in[0,1] .
$$

An $\alpha$-solution $x_{\alpha}(\cdot)$ of (4) is understood to be an absolutely continuous function $x_{\alpha}:[0, T] \rightarrow R^{n}$ which satisfies (5) almost everywhere. Let $X_{\alpha}$ denote the $\alpha$-solution set of (5) and let $X_{\alpha}(t)=\left\{x_{\alpha}(t): x_{\alpha}(\cdot) \in X_{\alpha}\right\}$. Clearly, a family of subsets $X_{t}=\left\{X_{\alpha}(t): \alpha \in[0,1]\right\}$ cannot satisfy the conditions of Theorem 2 (see $[5,6,9])$.

Therefore, we will consider an $R$-solution of fuzzy differential inclusion (4).
Definition 3. The upper semicontinuous fuzzy mapping $X$ : $[0, T] \rightarrow E^{n}$ which satisfies the system

$$
\begin{aligned}
& \sup _{\alpha \in[0,1]} h\left([X(t+\sigma)]^{\alpha}, \bigcup_{x \in[X(t)]^{\alpha}}\left\{x+\int_{t}^{t+\sigma}[F(s, x)]^{\alpha} \mathrm{d} s\right\}\right) \\
& =o(\sigma), \quad X(0)=X_{0}
\end{aligned}
$$

is called the $R$-solution $X(\cdot)$ of differential inclusion (4), where $\lim _{\sigma \rightarrow 0_{+}}(o(\sigma) / \sigma)=0$.

Theorem 4. Suppose that the following conditions hold:

(1) fuzzy mapping $F(\cdot, x)$ is measurable, for all $x \in R^{n}$;

(2) there exists $\lambda>0$ such that, for all $x^{\prime}, x^{\prime \prime} \in R^{n}$,

$$
D\left(F\left(t, x^{\prime}\right), F\left(t, x^{\prime \prime}\right)\right) \leq \lambda\left\|x^{\prime}-x^{\prime \prime}\right\|,
$$

for almost every $t \in[0, T]$;

(3) there exists $\gamma>0$ such that $D(F(t, x), \widehat{0}) \leq \gamma$, for almost every $t \in[0, T]$ and every $x \in R^{n}$;

(4) for all $\beta \in[0,1], x^{\prime}, x^{\prime \prime} \in R^{n}$ and almost every $t \in$ $[0, T]$

$$
\beta F\left(t, x^{\prime}\right)+(1-\beta) F\left(t, x^{\prime \prime}\right) \subset F\left(t, \beta x^{\prime}+(1-\beta) x^{\prime \prime}\right) .
$$

Then, there exists a unique $R$-solution $X(\cdot)$ of fuzzy system (4) defined on the interval $[0, \tau] \subseteq[0, T]$.

Proof. Let $S_{r}\left(X_{0}\right)=\left\{X \in E^{n}: D\left(X, X_{0}\right) \leq r\right\}$ and $\tau=$ $\min \{T, r / \gamma\}$.

By $[5,6]$, it follows that a family of subsets $X_{t}=\left\{X_{\alpha}(t)\right.$ : $\alpha \in[0,1]\}$ satisfy the conditions of Theorem 2 ; that is, $X_{t} \in$ $E^{n}$, for every $t \in[0, \tau]$.

Divide the interval $[0, \tau]$ into partial intervals by the points $t_{k}^{P}=k \tau 2^{-p}, k=0, \ldots, P, P=2^{p}, p \in N$. We use Euler algorithm; let the mapping $X^{P}(\cdot)$ be given by

$$
\left[X^{P}(t)\right]^{\alpha}=\bigcup_{x \in\left[X\left(t_{k}^{P}\right)\right]^{\alpha}}\left\{x+\int_{t_{k}^{P}}^{t}[F(s, x)]^{\alpha} \mathrm{d} s\right\},
$$

where $t \in\left[t_{k}^{P}, t_{k+1}^{P}\right], k \in\{0, \ldots, P\}, X(0)=X_{0}, \alpha \in[0,1]$.

By $[7,28]$, it follows that the sequence $\left\{\left[X^{P}(\cdot)\right]^{\alpha}\right\}_{p=1}^{\infty}$ is equicontinuous and fundamental and its limit is a unique $R$ solution $[X(\cdot)]^{\alpha}$ of differential inclusion (5) and $[X(t)]^{\alpha}=$ $X_{\alpha}(t)$, for every $t \in[0, \tau]$ and $\alpha \in[0,1]$. This concludes the proof.

Also, we consider the differential inclusion

$$
\dot{y} \in G(t, y), \quad y(0) \in Y_{0} \text {, }
$$

where $y \in R^{n}, t \in[0, T] \subset R_{+}, F:[0, T] \times R^{n} \rightarrow E^{n}, Y_{0} \in E^{n}$. 
Lemma 5. Let $F(t, x)$ and $G(t, y)$ satisfy conditions (1)-(4) of Theorem 4 and there exist $\eta>0$ and $\mu>0$ such that

$$
\begin{gathered}
D\left(\int_{t_{1}}^{t_{2}} F(s, x) d s, \int_{t_{1}}^{t_{2}} G(s, x) d s\right)<\eta\left(t_{2}-t_{1}\right), \\
D\left(X_{0}, Y_{0}\right)<\mu
\end{gathered}
$$

for every $x \in R^{n}$ and $t_{2}>t_{1}, t_{1}, t_{2} \in[0, T]$. $[0, T]$.

Then $D(X(t), Y(t)) \leq \mu e^{\lambda t}+(\eta / \lambda)\left(e^{\lambda t}-1\right)$, for every $t \epsilon$

Proof. Divide the interval $[0, T]$ into partial intervals by the points $t_{k}^{m}=k \Delta, \Delta=(T / m), k=0, \ldots, m, m \in N$. By Definition 3, we have

$$
\begin{aligned}
& D(X(t), Y(t)) \\
& \leq \sup _{\alpha \in[0,1]} h\left(\bigcup_{x \in\left[X\left(t_{k}\right)\right]^{\alpha}}\left\{x+\int_{t_{k}}^{t}[F(s, x)]^{\alpha} \mathrm{d} s\right\},\right. \\
& \left.\bigcup_{y \in\left[Y\left(t_{k}\right)\right]^{\alpha}}\left\{y+\int_{t_{k}}^{t}[G(s, y)]^{\alpha} \mathrm{d} s\right\}\right) \\
& +o(\Delta) \\
& \leq \sup _{\alpha \in[0,1]} h\left(\left[X\left(t_{k}\right)\right]^{\alpha}+\int_{t_{k}}^{t}\left[F\left(s,\left[X\left(t_{k}\right)\right]^{\alpha}\right)\right]^{\alpha} \mathrm{d} s,\right. \\
& \left.\left[Y\left(t_{k}\right)\right]^{\alpha}+\int_{t_{k}}^{t}\left[G\left(s,\left[Y\left(t_{k}\right)\right]^{\alpha}\right)\right]^{\alpha} \mathrm{d} s\right) \\
& +o(\Delta) \\
& \leq \sup _{\alpha \in[0,1]} h\left(\left[X\left(t_{k}\right)\right]^{\alpha}+\int_{t_{k}}^{t}\left[F\left(s,\left[X\left(t_{k}\right)\right]^{\alpha}\right)\right]^{\alpha} \mathrm{d} s,\right. \\
& \left.\left[X\left(t_{k}\right)\right]^{\alpha}+\int_{t_{k}}^{t}\left[F\left(s,\left[Y\left(t_{k}\right)\right]^{\alpha}\right)\right]^{\alpha} \mathrm{d} s\right) \\
& +\sup _{\alpha \in[0,1]} h\left(\left[X\left(t_{k}\right)\right]^{\alpha}+\int_{t_{k}}^{t}\left[F\left(s,\left[Y\left(t_{k}\right)\right]^{\alpha}\right)\right]^{\alpha} \mathrm{d} s,\right. \\
& \left.\left[Y\left(t_{k}\right)\right]^{\alpha}+\int_{t_{k}}^{t}\left[F\left(s,\left[Y\left(t_{k}\right)\right]^{\alpha}\right)\right]^{\alpha} \mathrm{d} s\right) \\
& +\sup _{\alpha \in[0,1]} h\left(\left[Y\left(t_{k}\right)\right]^{\alpha}+\int_{t_{k}}^{t}\left[F\left(s,\left[Y\left(t_{k}\right)\right]^{\alpha}\right)\right]^{\alpha} \mathrm{d} s,\right. \\
& \left.\left[Y\left(t_{k}\right)\right]^{\alpha}+\int_{t_{k}}^{t}\left[G\left(s,\left[Y\left(t_{k}\right)\right]^{\alpha}\right)\right]^{\alpha} \mathrm{d} s\right)
\end{aligned}
$$$$
+o(\Delta)
$$

$$
\begin{aligned}
& \leq \sup _{\alpha \in[0,1]} h\left(\int_{t_{k}}^{t}\left[F\left(s,\left[X\left(t_{k}\right)\right]^{\alpha}\right)\right]^{\alpha} \mathrm{d} s,\right. \\
& \left.\quad \int_{t_{k}}^{t}\left[F\left(s,\left[Y\left(t_{k}\right)\right]^{\alpha}\right)\right]^{\alpha} \mathrm{d} s\right) \\
& +\sup _{\alpha \in[0,1]} h\left(\left[X\left(t_{k}\right)\right]^{\alpha},\left[Y\left(t_{k}\right)\right]^{\alpha}\right) \\
& \leq \sup _{\alpha \in[0,1]} h\left(\int_{t_{k}}^{t}\left[F\left(s,\left[X\left(t_{k}\right)\right]^{\alpha}\right)\right]^{\alpha} \mathrm{d} s,\right. \\
& \left.\quad \int_{t_{k}}^{t}\left[G\left(s,\left[Y\left(t_{k}\right)\right]^{\alpha}\right)\right]^{\alpha} \mathrm{d} s\right)+o(\Delta) \\
& \leq \int_{t_{k}}^{t} \lambda D\left(X\left(t_{k}\right), Y\left(t_{k}\right)\right) \mathrm{d} s \\
& \quad+D\left(X\left(t_{k}\right), Y\left(t_{k}\right)\right)+\eta\left(t-t_{k}\right)+o(\Delta) \\
& \leq\left(\left(t-t_{k}\right) \lambda+1\right) D\left(X\left(t_{k}\right), Y\left(t_{k}\right)\right) \\
& \quad+\eta\left(t-t_{k}\right)+o(\Delta) \leq \mu e^{\lambda t}+\frac{\eta}{\lambda}\left(e^{\lambda t}-1\right)
\end{aligned}
$$

for every $t \in[0, T]$. This concludes the proof.

Remark 6. If $X_{0}=Y_{0}$, then $D(X(t), Y(t)) \leq(\eta / \lambda)\left(e^{\lambda t}-1\right)$, for every $t \in[0, T]$.

\section{The Method of Partial Averaging}

Now, consider the fuzzy differential inclusion with a small parameter

$$
\dot{x} \in \varepsilon F(t, x), \quad x(0) \in X_{0},
$$

where $X \in R^{n}, t \in R_{+}, F: R_{+} \times R^{n} \rightarrow E^{n}, X_{0} \in E^{n}$, and $\varepsilon>0$ is a small parameter.

In this work, we associate the following partial averaged fuzzy differential inclusion with the inclusion (10):

$$
\dot{y} \in \varepsilon G(t, y), \quad y(0) \in X_{0},
$$

where $G: R_{+} \times R^{n} \rightarrow E^{n}$ such that

$$
\lim _{T \rightarrow \infty} D\left(\frac{1}{T} \int_{0}^{T} F(t, x) \mathrm{d} t, \frac{1}{T} \int_{0}^{T} G(t, x) \mathrm{d} t\right)=0 .
$$

Theorem 7. Let in domain $Q=\{(t, x): t \geq 0, x \in D \in$ $\left.\operatorname{conv}\left(R^{n}\right)\right\}$ the following conditions hold:

(1) mappings $F(\cdot, x), G(\cdot, x)$ are measurable on $R_{+}$;

(2) mappings $F(t, \cdot), G(t, \cdot)$ satisfy a Lipschitz condition

$$
\begin{aligned}
& D\left(F\left(t, x^{\prime}\right), F\left(t, x^{\prime \prime}\right)\right) \leq \lambda\left\|x^{\prime}-x^{\prime \prime}\right\|, \\
& D\left(G\left(t, x^{\prime}\right), G\left(t, x^{\prime \prime}\right)\right) \leq \lambda\left\|x^{\prime}-x^{\prime \prime}\right\|,
\end{aligned}
$$

with a Lipschitz constant $\lambda>0$; 
(3) there exists $\gamma>0$ such that

$$
D(F(t, x), \widehat{0}) \leq \gamma, \quad D(G(t, x), \widehat{0}) \leq \gamma
$$

for almost every $t \in[0, T]$ and every $x \in R^{n}$;

(4) for all $\beta \in[0,1], x^{\prime}, x^{\prime \prime} \in R^{n}$ and almost every $t \in$ $[0, T]$,

$$
\begin{aligned}
& \beta F\left(t, x^{\prime}\right)+(1-\beta) F\left(t, x^{\prime \prime}\right) \\
& \subset F\left(t, \beta x^{\prime}+(1-\beta) x^{\prime \prime}\right), \\
& \beta G\left(t, x^{\prime}\right)+(1-\beta) G\left(t, x^{\prime \prime}\right) \\
& \subset G\left(t, \beta x^{\prime}+(1-\beta) x^{\prime \prime}\right) .
\end{aligned}
$$

(5) limit (15) exists uniformly with respect to $x$ in the domain $G$;

(6) for any $X_{0}\left(\left[X_{0}\right]^{0} \subset D^{\prime} \subset D\right), \varepsilon \in(0, v]$, and $t>0$, the R-solution of the inclusion (10) together with a $\rho$-neighborhood belongs to the domain $G$; that is, $[X(t)]^{0}+S_{\rho}(0) \subset D$, for every $t>0$.

Then, for any $\eta \in(0, \rho]$ and $L>0$, there exists $\varepsilon_{0}(\eta, L)>0$ such that, for all $\varepsilon \in\left(0, \varepsilon_{0}\right]$ and $t \in\left[0, L \varepsilon^{-1}\right]$, the following inequality holds:

$$
D(X(t), Y(t))<\eta
$$

where $X(\cdot), Y(\cdot)$ are the $R$-solutions of initial and partial averaged inclusions.

Proof. Divide the interval $\left[0, L \varepsilon^{-1}\right]$ on the partial intervals by the points $t_{k}=(k L / \varepsilon m), k \in\{0,1, \ldots, m-1\}$. We denote fuzzy mappings $X^{m}(\cdot)$ and $Y^{m}(\cdot)$ such that

$$
\begin{array}{r}
{\left[X^{m}(t)\right]^{\alpha}=\bigcup_{x \in\left[X\left(t_{k}\right)\right]^{\alpha}}\left\{x+\varepsilon \int_{t_{k}}^{t}[F(s, x)]^{\alpha} \mathrm{d} s\right\},} \\
{\left[X^{m}(0)\right]^{\alpha}=\left[X_{0}\right]^{\alpha},} \\
{\left[Y^{m}(t)\right]^{\alpha}=\bigcup_{y \in\left[Y\left(t_{k}\right)\right]^{\alpha}}\left\{y+\varepsilon \int_{t_{k}}^{t}[G(s, y)]^{\alpha} \mathrm{d} s\right\},} \\
{\left[Y^{m}(0)\right]^{\alpha}=\left[X_{0}\right]^{\alpha},}
\end{array}
$$

for every $\alpha \in[0,1], t \in\left[t_{k}, t_{k+1}\right], k \in\{0,1, \ldots, m-1\}$.
Then

$$
\begin{aligned}
& D\left(X^{m}\left(t_{k}\right), X\left(t_{k}\right)\right) \\
& \leq \sup _{\alpha \in[0,1]} h\left(\bigcup_{x \in\left[X^{m}\left(t_{k-1}\right)\right]^{\alpha}}\left\{x+\varepsilon \int_{t_{k-1}}^{t_{k}}[F(s, x)]^{\alpha} \mathrm{d} s\right\},\right. \\
& \left.\bigcup_{x \in\left[X\left(t_{k-1}\right)\right]^{\alpha}}\left\{x+\varepsilon \int_{t_{k-1}}^{t_{k}}[F(s, x)]^{\alpha} \mathrm{d} s\right\}\right) \\
& \leq\left(1+\varepsilon\left(t_{k}-t_{k-1}\right)\right. \\
& \left.+o\left(t_{k}-t_{k-1}\right) \lambda\right) D\left(X^{m}\left(t_{k-1}\right), X\left(t_{k-1}\right)\right) \\
& t_{k}-t_{k-1}
\end{aligned}
$$

Also, we take

$$
D\left(Y^{m}\left(t_{k}\right), Y\left(t_{k}\right)\right) \leq \frac{o\left(t_{k}-t_{k-1}\right)}{t_{k}-t_{k-1}}\left(e^{\lambda L}-1\right) .
$$

$$
\begin{aligned}
& \text { As for } t \in\left[t_{k}, t_{k+1}\right], \\
& D\left(X^{m}(t), X^{m}\left(t_{k}\right)\right) \\
& \leq \sup _{\alpha \in[0,1]} h \\
& \quad \times\left(\bigcup_{x \in\left[X^{m}\left(t_{k}\right)\right]^{\alpha}}\left\{x+\varepsilon \int_{t_{k}}^{t}[F(s, x)]^{\alpha} \mathrm{d} s\right\},\left[X^{m}\left(t_{k}\right)\right]^{\alpha}\right) \\
& \leq \varepsilon \gamma\left(t-t_{k}\right) \leq \frac{\gamma L}{m},
\end{aligned}
$$

$$
D\left(Y^{m}(t), Y^{m}\left(t_{k}\right)\right) \leq \varepsilon \gamma\left(t-t_{k}\right) \leq \frac{\gamma L}{m} .
$$

Using estimates (22)-(25), for any $\eta>0$, there exists $m_{0}$ such that, for $m>m_{0}$, we have

$$
\begin{aligned}
& D\left(X^{m}(t), X(t)\right) \leq \frac{\eta}{4} \\
& D\left(Y^{m}(t), Y(t)\right) \leq \frac{\eta}{4} .
\end{aligned}
$$

Taking into account Lemma 5, for any $v>0$, there exists $\varepsilon_{0}>0$ such that, for all $\varepsilon \in\left(0, \varepsilon_{0}\right]$, the following inequality holds:

$$
D\left(X^{m}\left(t_{k+1}\right), Y\left(t_{k+1}\right)\right) \leq \frac{\nu}{\lambda}\left(e^{\lambda L}-1\right) .
$$

By combining (26) and (27) and choosing $m \geq$ $\max \left\{m_{0}, 8 \gamma L / \eta\right\}$ and $\nu<\left(\eta \lambda / 4\left(e^{\lambda L}-1\right)\right)$, we obtain (19). The theorem is proved. 


\section{Conclusion}

If $F(\cdot, x)$ is continuous on $[0, T]$, then, instead of $(5)$, it is possible to consider the following more simple equation:

$$
\begin{aligned}
\sup _{\alpha \in[0,1]} & h\left([X(t+\sigma)]^{\alpha}, \bigcup_{x \in[X(t)]^{\alpha}}\left\{x+\sigma[F(t, x)]^{\alpha}\right\}\right) \\
= & o(\sigma), \quad X(0)=X_{0},
\end{aligned}
$$

and, similarly, we can prove all the results received earlier.

\section{Conflict of Interests}

The authors declare that there is no conflict of interests regarding the publication of this paper.

\section{References}

[1] J.-P. Aubin, "Fuzzy differential inclusions," Problems of Control and Information Theory, vol. 19, no. 1, pp. 55-67, 1990.

[2] V. A. Baidosov, "Differential inclusions with fuzzy right-hand side," Soviet Mathematics, vol. 40, no. 3, pp. 567-569, 1990.

[3] V. A. Baidosov, "Fuzzy differential inclusions," Journal of Applied Mathematics and Mechanics, vol. 54, no. 1, pp. 8-13, 1990.

[4] E. Hüllermeier, "Towards modelling of fuzzy functions," in Proceedings of the 3rd European Congress on Intelligent Techniques and Soft Computing (EUFIT '95), pp. 150-154, 1995.

[5] E. Hüllermeier, "An approach to modelling and simulation of uncertain dynamical system," International Journal of Uncertainty, Fuzziness and Knowledge-Based Systems, vol. 7, pp. 117137, 1997.

[6] E. Hüllermeier, "A fuzzy simulation method," in Proceedings of the 1st International ICSC Symposium on Intelligent Industrial Automation (IIA '96) and Soft Computing (SOCO '96), Reading, UK, March 1996.

[7] A. I. Panasyuk and V. I. Panasyuk, "An equation generated by a differential inclusion," Mathematical Notes of the Academy of Sciences of the USSR, vol. 27, no. 3, pp. 213-218, 1980.

[8] S. Abbasbandy, T. A. Viranloo, Ó. López-Pouso, and J. J. Nieto, "Numerical methods forfuzzy differential inclusions," Computers \& Mathematics with Applications, vol. 48, no. 10-11, pp. 1633-1641, 2004.

[9] R. P. Agarwal, D. O’Regan, and V. Lakshmikantham, "A stacking theorem approach for fuzzy differential equations," Nonlinear Analysis: Theory, Methods and Applications, vol. 55, no. 3, pp. 299-312, 2003.

[10] R. P. Agarwal, D. O’Regan, and V. Lakshmikantham, "Maximal solutions and existence theory for fuzzy differential and integral equations," Journal of Applied Analysis, vol. 11, no. 2, pp. 171-186, 2005.

[11] P. L. Antonelli and V. Křivan, "Fuzzy differential inclusions as substitutes for stochastic differential equations in population biology," Open Systems \& Information Dynamics, vol. 1, no. 2, pp. 217-232, 1992.

[12] M. Chen, D. Li, and X. Xue, "Periodic problems of first order uncertain dynamical systems," Fuzzy Sets and Systems, vol. 162, no. 1, pp. 67-78, 2011.

[13] M. Chen and C. Han, "Periodic behavior of semi-linear uncertain dynamical systems," Fuzzy Sets and Systems, vol. 230, no. 1, pp. 82-91, 2013.
[14] G. Colombo and V. Krivan, "Fuzzy differential inclusions and nonprobabilistic likelihood," Dynamic Systems and Applications, vol. 1, pp. 419-440, 1992.

[15] M. Guo, X. Xue, and R. Li, "Impulsive functional differential inclusions and fuzzy population models," Fuzzy Sets and Systems, vol. 138, no. 3, pp. 601-615, 2003.

[16] V. Laksmikantham, "Set differential equations versus fuzzy differential equations," Applied Mathematics and Computation, vol. 164, no. 2, pp. 277-297, 2005.

[17] V. Lakshmikantham, T. Granna Bhaskar, and J. Vasundhara Devi, Theory of Set Differential Equations in Metric Spaces, Cambridge Scientific Publishers, Cambridge, UK, 2006.

[18] V. Lakshmikantham and R. N. Mohapatra, Theory of Fuzzy Differential Equations and Inclusions, Taylor \& Francis, London, UK, 2003.

[19] D. Li, M. Chen, and X. Xue, "Two-point boundary value problems of uncertain dynamical systems," Fuzzy Sets and Systems, vol. 179, no. 1, pp. 50-61, 2011.

[20] K. K. Majumdar and D. D. Majumder, "Fuzzy differential inclusions in atmospheric and medical cybernetics," IEEE Transactions on Systems, Man, and Cybernetics B: Cybernetics, vol. 34, no. 2, pp. 877-887, 2004.

[21] A. V. Plotnikov, T. A. Komleva, and L. I. Plotnikova, "The partial averaging of differential inclusions with fuzzy right-hand side," Journal of Advanced Research in Dynamical and Control Systems, vol. 2, no. 2, pp. 26-34, 2010.

[22] A. V. Plotnikov, T. A. Komleva, and L. I. Plotnikova, "On the averaging of differential inclusions with fuzzy right-hand side when the average of the right-hand side is absent," Iranian Journal of Optimization, vol. 2, no. 3, pp. 506-517, 2010.

[23] S. Klymchuk, A. Plotnikov, and N. Skripnik, "Overview of V.A. Plotnikov's research on averaging of differential inclusions," Physica D: Nonlinear Phenomena, vol. 241, no. 22, pp. 1932-1947, 2012.

[24] N. A. Perestyuk, V. A. Plotnikov, A. M. Samoŭlenko, and N. V. Skripnik, Differential Equations with Impulse Effects. Multivalued Right-Hand Sides with Discontinuities, vol. 40 of de Gruyter Studies in Mathematics, Walter de Gruyter, Berlin, Germany, 2011.

[25] V. A. Plotnikov, "Averaging of differential inclusions," Ukrainian Mathematical Journal, vol. 31, no. 5, pp. 454-457, 1979.

[26] V. A. Plotnikov, "Partial averaging of differential inclusions," Mathematical Notes of the Academy of Sciences of the USSR, vol. 27, no. 6, pp. 456-459, 1980.

[27] V. A. Plotnikov, The Averaging Method in Control Problems, Lybid, Kiev, Ukraine, 1992.

[28] V. A. Plotnikov, A. V. Plotnikov, and A. N. Vityuk, Differential Equations with a Multivalued Right-Hand Side, Asymptotic Methods, AstroPrint, Odessa, Ukraine, 1999.

[29] C. V. Negoita and D. A. Ralescu, Application of Fuzzy Sets to Systems Analysis, John Wiley \& Sons, New York, NY, USA, 1975.

[30] M. L. Puri and D. A. Ralescu, "Fuzzy random variables," Journal of Mathematical Analysis and Applications, vol. 114, no. 2, pp. 409-422, 1986. 


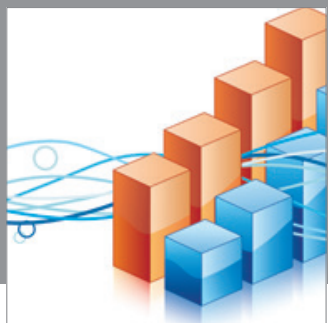

Advances in

Operations Research

mansans

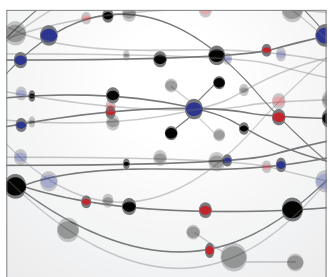

The Scientific World Journal
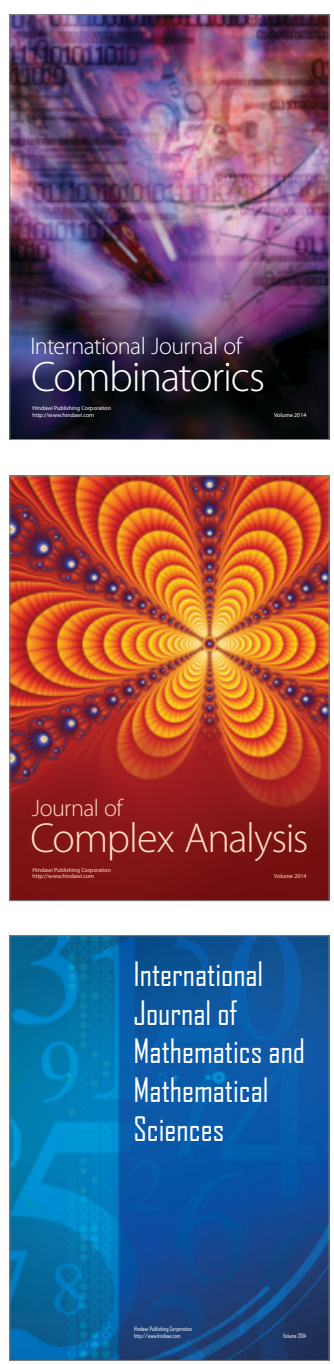
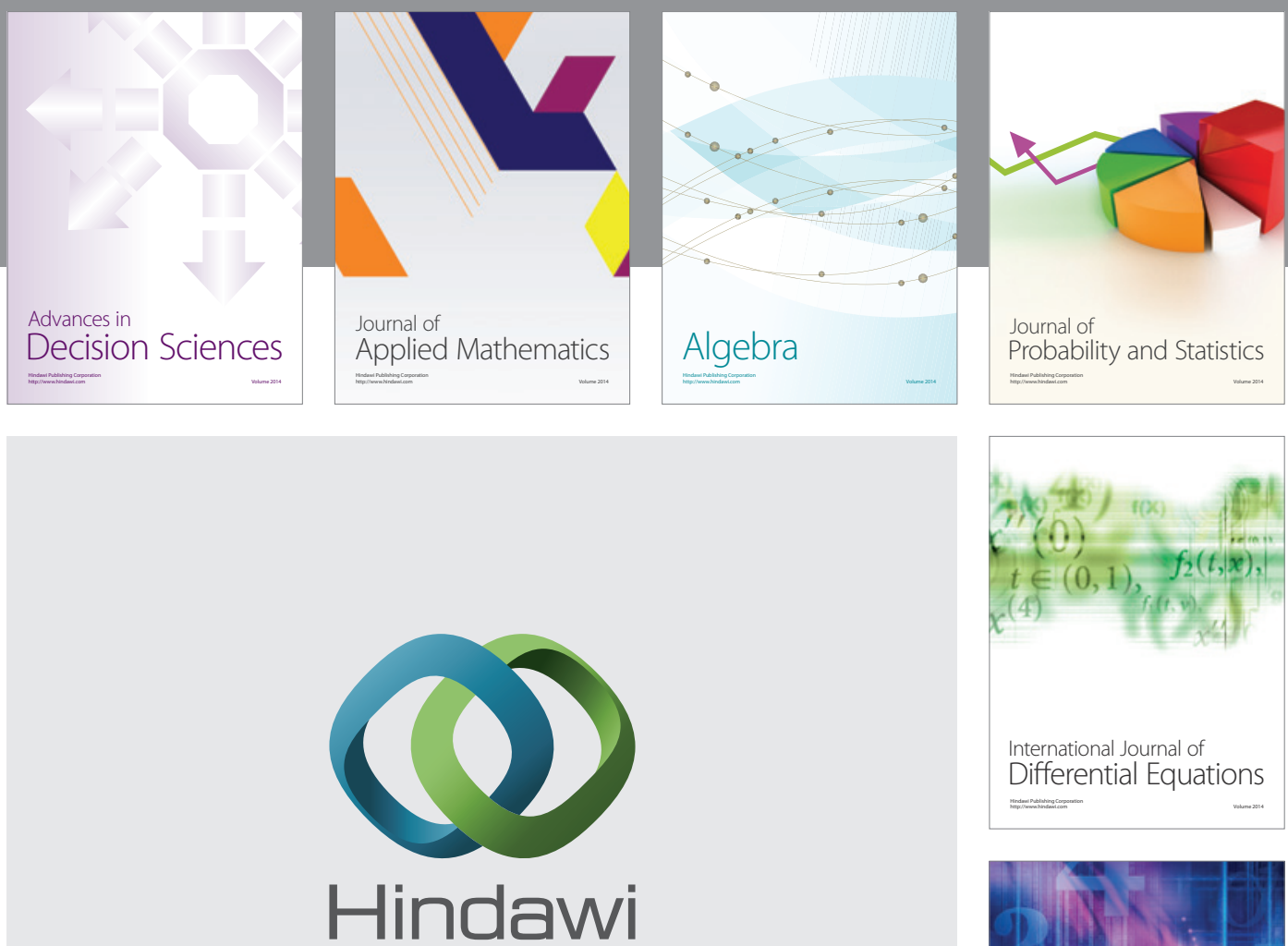

Submit your manuscripts at http://www.hindawi.com
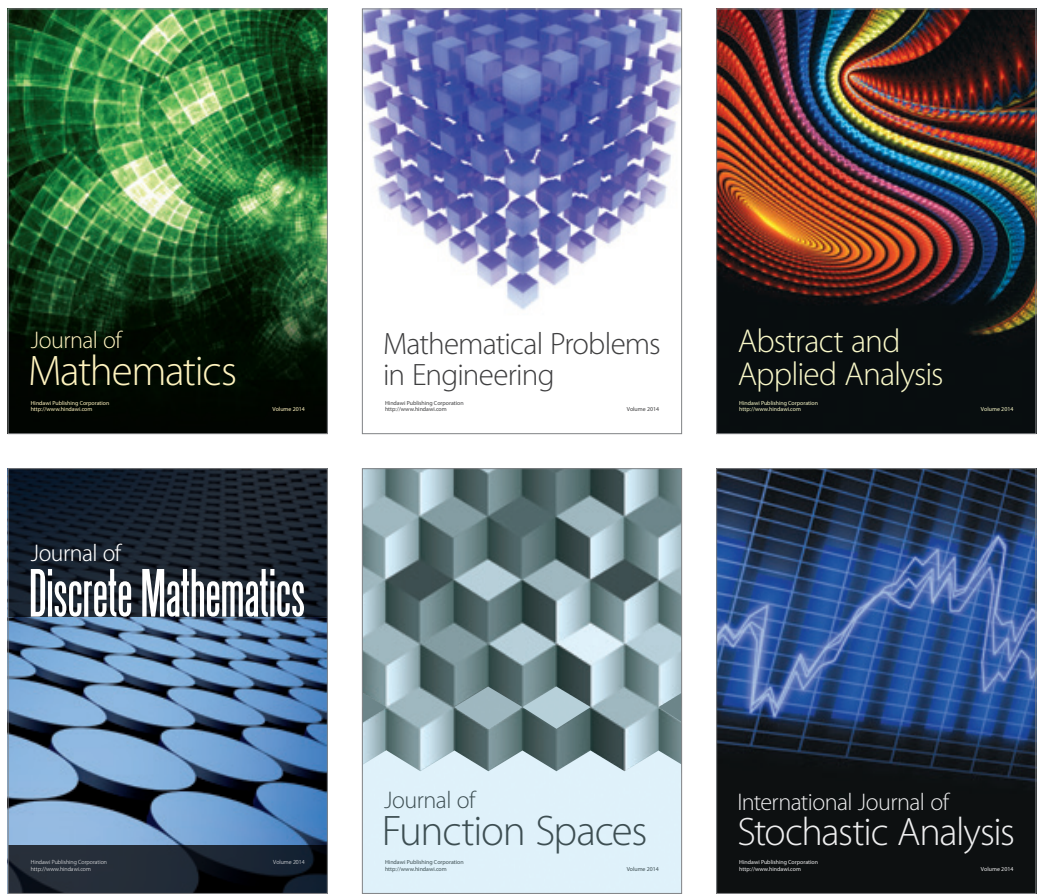

Journal of

Function Spaces

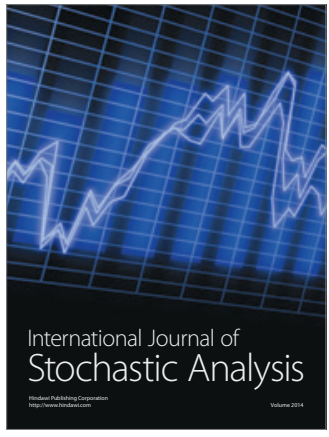

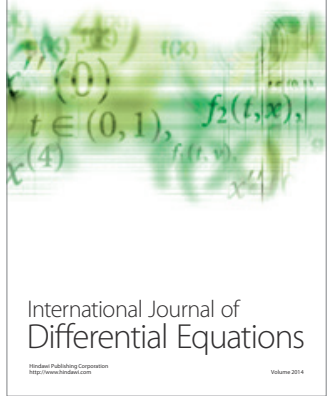
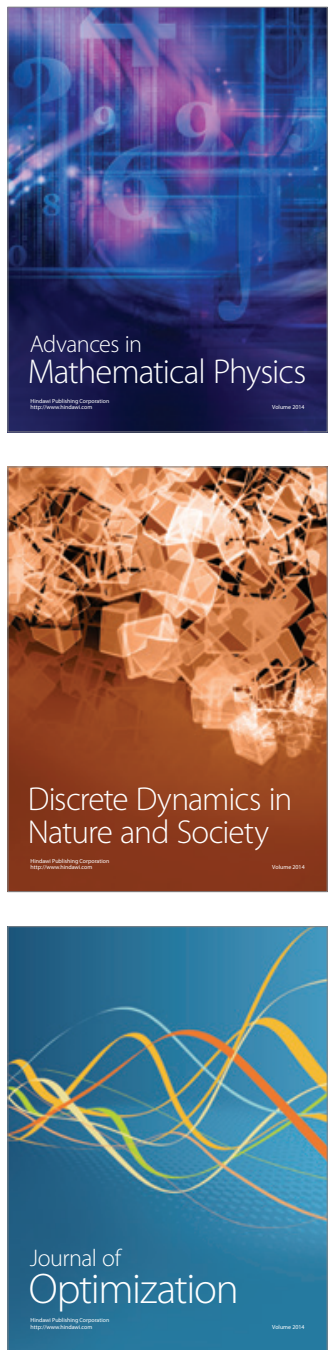